\title{
Deforming the Hippocampal Map
}

\author{
David S. Touretzky, ${ }^{1 *}$ Wendy E. Weisman, ${ }^{2}$ Mark C. Fuhs, ${ }^{1}$ William E. Skaggs, ${ }^{3}$ \\ Andre A. Fenton, ${ }^{4,5}$ and Robert U. Muller ${ }^{5,6}$
}

\begin{abstract}
To investigate conjoint stimulus control over place cells, Fenton et al. (J Gen Physiol 116:191-209, 2000a) recorded while rats foraged in a cylinder with $45^{\circ}$ black and white cue cards on the wall. Card centers were $135^{\circ}$ apart. In probe trials, the cards were rotated together or apart by $25^{\circ}$. Firing field centers shifted during these trials, stretching and shrinking the cognitive map. Fenton et al. (2000b) described this deformation with an ad hoc vector field equation. We consider what sorts of neural network mechanisms might be capable of accounting for their observations. In an abstract, maximum likelihood formulation, the rat's location is estimated by a conjoint probability density function of landmark positions. In an attractor neural network model, recurrent connections produce a bump of activity over a two-dimensional array of cells; the bump's position is influenced by landmark features such as distances or bearings. If features are chosen with appropriate care, the attractor network and maximum likelihood models yield similar results, in accord with previous demonstrations that recurrent neural networks can efficiently implement maximum likelihood computations (Pouget et al. Neural Comput 10:373-401, 1998; Deneve et al. Nat Neurosci 4:826-831, 2001). $\odot 2004$ Wiley-Liss, Inc.
\end{abstract}

KEY WORDS: hippocampal map; attractor network model; maximum likelihood estimation

\section{INTRODUCTION}

Many cells in the rat hippocampus exhibit spike activity focused on times when the rat is located at specific places in the environment. Dozens of studies over the past three decades have demonstrated that these firing field locations can be controlled by visual landmarks. The studies have yielded enough quantitative information to enable development of preliminary computational models of these "place cell" responses. Currently, however,

${ }^{1}$ Computer Science Department and Center for the Neural Basis of Cognition, Carnegie Mellon University, Pittsburgh, Pennsylvania; ${ }^{2}$ Saint Paul, Minnesota; ${ }^{3}$ Arizona Research Laboratories, Division of Neural Systems, Memory, and Aging, Tucson, Arizona; ${ }^{4}$ Institute of Physiology, Academy of Sciences of the Czech Republic, Prague, Czech Republic; ${ }^{5}$ Department of Physiology and Pharmacology, State University of New York Health Sciences Center at Brooklyn, Brooklyn, New York; ${ }^{6}$ Medical Research Council Center for Synaptic Plasticity, Department of Anatomy, University of Bristol, Bristol, United Kingdom

Grant sponsor: National Institutes of Health; Grant number: MH59932; NS20686; Grant number: NS37150; Grant sponsor: National Science Foundation; Grant numbers: IIS-9978403; DGE-9987588; Grant sponsor: Medical Research Council.

*Correspondence to: David S. Touretzky, Computer Science Department, Carnegie Mellon University, Pittsburgh, PA 15213-3891.

E-mail: dst@cs.cmu.edu

Accepted for publication 9 April 2004

DOI 10.1002/hipo.20029

Published online 8 June 2004 in Wiley InterScience (www.interscience. wiley.com). the pool of candidate models is very diverse, and there is not even consensus on the general form of a correct model, much less the detailed values of parameters (see Hippocampus 9:4, Special Issue on Place Cells, for examples and references). In an effort to narrow down the pool of viable models, some of the most useful information has come from experiments that have manipulated features of the environment and quantified the resulting changes in spatial firing properties of hippocampal cells. One set of particularly informative findings comes from a recent study by Fenton et al. (2000a) on the consequences of changing the angular separation between two cue cards mounted on the walls of a cylindrical arena. The authors presented a mathematical model of the deformations they observed in the "cognitive map," but it was purely descriptive; there was no claim that the hippocampus actually derived firing fields this way. The aim of the current study is to consider what sorts of neural network mechanisms might be capable of accounting for their observations.

After reviewing the Fenton et al. model, we develop a theoretically justified account of the map deformation effect by constructing a new model based on maximum likelihood estimation. We then present a third model, an attractor neural network, that approximates the behavior of the maximum likelihood model as a computation that could be implemented in the hippocampus. Our results are in accord with previous observations that recurrent neural networks can efficiently implement maximum likelihood estimation (Pouget et al., 2002).

\section{THE EXPERIMENT}

To investigate conjoint stimulus control over place cells, Fenton et al. (2000a) recorded while rats foraged in a cylinder with one white and one black cue card on the wall. The cards each subtended $45^{\circ}$ of arc, and their centers were $135^{\circ}$ apart, leaving a gap of $90^{\circ}$ between the right edge of the white card and the left edge of the black card. In probe trials the cards were rotated to increase or decrease their separation by $25^{\circ}$. Firing field centers shifted systematically during these trials, distorting the cognitive map. In contrast, on trials in which one card was removed and the other rotated, the map did not distort, but rotated with the remaining card, demonstrating its continuing salience and a nearly ideal form of pattern completion. 
Fenton et al. (2000b) presented a mathematical model of the deformations they observed in their experiments. The model described how they believed firing field centers moved as a result of rotating the cue cards or deleting a card: all cells were controlled by both cards, but to varying degrees based on the distance of the field center to each card. This finding contrasts with previous models, in which cells differ in their responses to environmental manipulations because they receive input from different subsets of cues (Shapiro et al. 1997; Hartley et al., 2000).

\section{REFORMULATED VECTOR FIELD MODEL}

The vector field model of Fenton et al. (2000b) had two parts: an angular component that determined how firing field centers rotate around the center of the arena, and a translational component that corrected a problem with the prediction of the rotation equation when the cards were moved together or apart. We begin by presenting this model in a slightly different formulation for improved clarity and completeness.

Our variant assumes that upon entry into the cylinder during a probe trial, the animal's head direction estimate is reset so that "east" is the direction defined by the line from the arena center to a reference point on the cylinder wall halfway between the closest edges of the two cue cards. If the cards rotate by opposite amounts (e.g., $+10^{\circ}$ and $-10^{\circ}$ ), their edges move closer together or farther apart, but there is no change in the reference point. If the cards rotate by identical amounts, the reference point rotates as well, so the change is undetectable by the model due to the resetting of the head direction system. If the cards rotate by unequal amounts, after head direction reset the model will see only their relative motion. Finally, if one card is removed, the reference point is defined to be in "standard" position with respect to the remaining card, meaning $45^{\circ}$ counterclockwise from the left edge of the black card or $45^{\circ}$ clockwise from the right edge of the white card and, again, any rotation of the card is undetectable.

Head direction reset restricts the model to operating in the reference frame defined by the cards. There is no provision for an external reference frame tied to the experimental chamber to influence place cell firing, in agreement with the finding that when the two cards were rotated together no influence of a room frame was seen. The head direction reset assumption greatly simplifies the vector field equations, ensuring that angular displacements of the cards with respect to the reference point are always equal and opposite. We will therefore adopt the convention that the white card rotates by an angle $\alpha$ and the black card by $-\alpha$.

The next step in our formulation is to calculate displacement vectors, in room coordinates, based on rotations of the individual cue cards. Ignoring the black card for the moment, if the white card rotates by an angle $\alpha$, then all firing fields influenced by this card should rotate by $\alpha$ (see Fig. 1). We represent each field by the location of its center. Assume the cylinder is centered at the origin, and let $\mathrm{R}$ be its radius. A field on the east edge of the cylinder, at location [R, 0], would rotate to a new position $[\mathrm{R} \cos \alpha, \mathrm{R} \sin \alpha$ ].

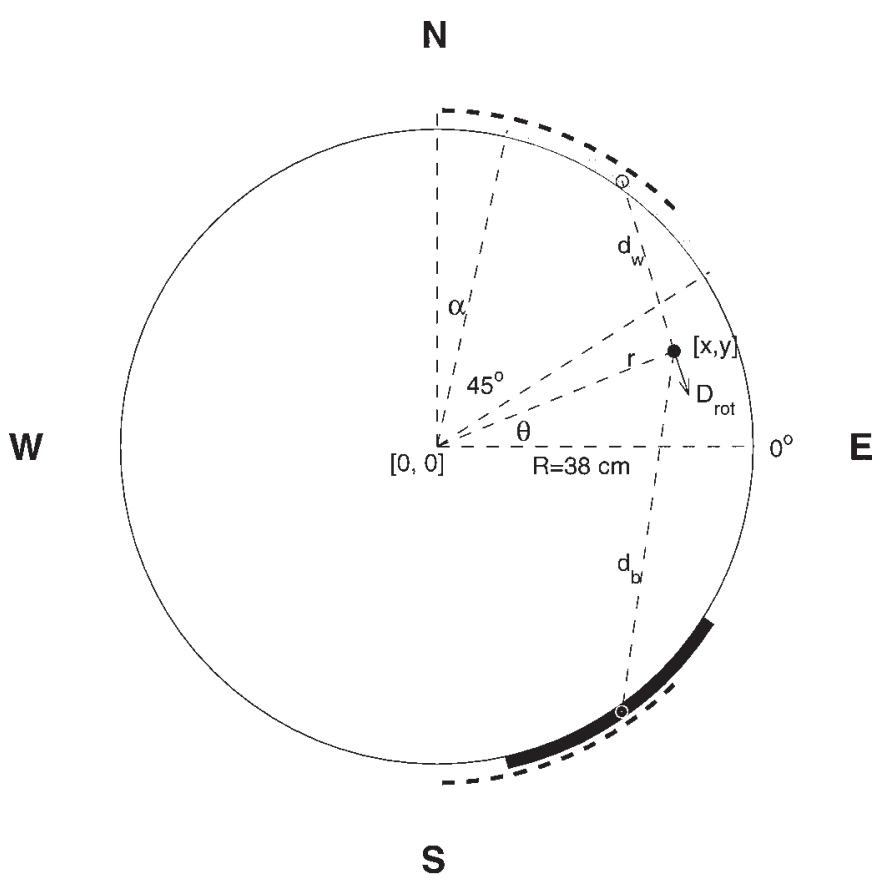

FIGURE 1. Calculation of the rotational displacement vector $D_{\text {rot }}$ for a point $[x, y]$ when the cards are rotated by $2 \alpha=-25^{\circ}$. Dotted arcs show cards in their standard position, solid arcs show rotated position.

Hence, the white card-dependent rotational displacement vector for this specific point would be:

$$
\mathrm{W}_{\mathrm{R}, 0}=\left[\begin{array}{c}
\mathrm{R} \cos \alpha \\
\mathrm{R} \sin \alpha
\end{array}\right]-\left[\begin{array}{l}
\mathrm{R} \\
0
\end{array}\right]=\mathrm{R} \cdot\left[\begin{array}{c}
\cos \alpha-1 \\
\sin \alpha
\end{array}\right]
$$

Any point on the $\mathrm{x}$-axis at a distance $r$ from the origin would have the same rotational displacement vector $W_{\mathrm{R}, 0}$, scaled by $\mathrm{r} / \mathrm{R}$. In the general case of an arbitrary point $[\mathrm{x}, \mathrm{y}]$ in the cylinder, at a distance $r=\sqrt{x^{2}+y^{2}}$ from the origin and an angular displacement $\theta=\operatorname{atan}(y / x)$ from the positive $x$-axis, the white card-dependent rotational displacement vector is given by:

$$
\mathrm{W}_{\mathrm{x}, \mathrm{y}}=\mathrm{r} \cdot\left[\begin{array}{cc}
\cos \theta & -\sin \theta \\
\sin \theta & \cos \theta
\end{array}\right] \times\left[\begin{array}{c}
\cos \alpha-1 \\
\sin \alpha
\end{array}\right]
$$

The black card-influenced rotational displacement vector $\mathrm{B}_{\mathrm{x}, \mathrm{y}}$ is calculated analogously using $-\alpha$. The overall rotational displacement of a point $[\mathrm{x}, \mathrm{y}]$ is the average of its white and black card rotational displacement vectors, weighted by relative distance to the two cards, so that the nearer card has proportionally greater influence. If $\mathrm{d}_{\mathrm{w}}$ and $\mathrm{d}_{\mathrm{b}}$ are the distances from the point $[\mathrm{x}, \mathrm{y}]$ to the centers of the white and black cards, respectively, then the net rotational displacement is

$$
D_{\text {rot }}=\frac{d_{w} B_{x, y}+d_{b} W_{x, y}}{d_{w}+d_{b}}
$$

The result of this equation is shown in the leftmost plot in Figure 2. This purely rotational displacement does not accurately 

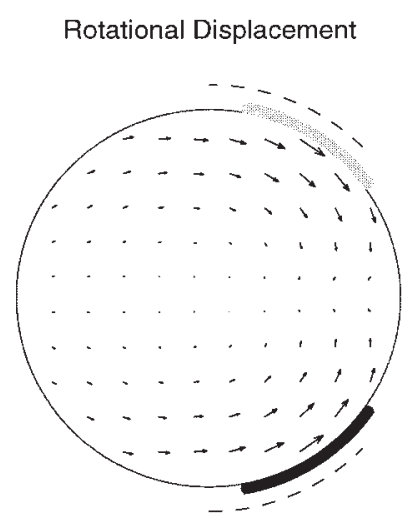
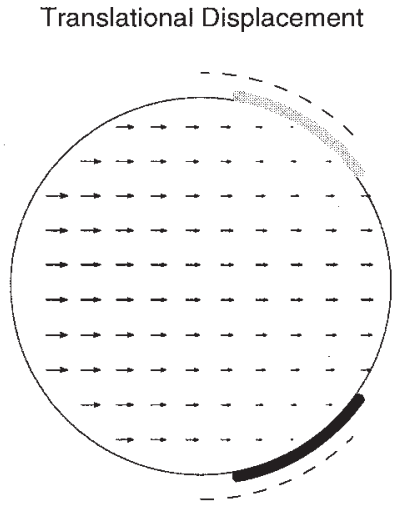

Total Displacement

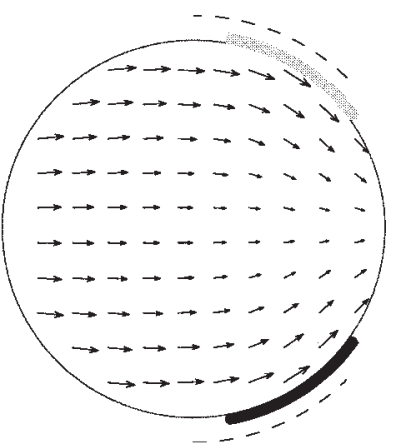

FIGURE 2. Calculated displacement vectors when cards are rotated closer together by $25^{\circ}$ : rotational displacement $D_{\text {rot }}$ from eq. (3), translational displacement $D_{\text {trans }}$ from eq. (4), and total displacement $\mathrm{D}_{\text {tot }}$ from eq. (5).

capture the distortion of relative field locations observed by Fenton et al. When the two cards move closer together, all field centers are displaced slightly toward the cards; when the cards move apart, field centers are displaced away from the cards. Fields close to a card show less translation, but more rotation, than fields distant from either card. Fenton et al. added a translational term to their equation to reproduce this effect. $\mathrm{Let}_{\mathrm{c}}$ be the rotational displacement vector denoting the movement of the white card center from its standard position to its current location. Let $\mathrm{B}_{\mathrm{c}}$ be the rotational displacement vector for the black card center. The translational displacement of a point at distances $d_{w}, d_{b}$ from the white and black cards, respectively, is defined as:

$$
\mathrm{D}_{\text {trans }}=\frac{\mathrm{W}_{\mathrm{c}}+\mathrm{B}_{\mathrm{c}}}{\mathrm{c}_{2} \cdot\left(\frac{1}{\mathrm{~d}_{\mathrm{w}}}+\frac{1}{\mathrm{~d}_{\mathrm{b}}}\right)}
$$

where $c_{2}=83.4 \mathrm{~cm}$ determines the dropoff of the translational term as distance to the cards decreases. Note that if either $\mathrm{d}_{\mathrm{w}}$ or $\mathrm{d}_{\mathrm{b}}$ is small, the denominator of the equation will be large, and the translational term will be attenuated relative to the rotational term.
But at locations distant from both cards, the translational term is significant. The contribution of this translational displacement term is shown in the center plot of Figure 2.

The total displacement of a point is the sum of its rotational and translational displacements, and is shown in the rightmost plot in Figure 2:

$$
\mathrm{D}_{\mathrm{tot}}=D_{\text {rot }}+\mathrm{D}_{\text {trans }}
$$

Figure 3 shows the rotational, translational, and total displacement vectors when the cards are moved apart by $25^{\circ}$. Once again, the translational component helps overcome the shortcomings of the rotational component at locations distant from both cue cards.

The Fenton et al. (2000b) model produces a good match to the experimental data of Fenton and Muller on cards moving together or apart by $25^{\circ}$. Furthermore, when one card is removed and the other card rotated, the model correctly predicts that fields will rotate without distortion, i.e., they rotate in the room frame to maintain their "standard" position relative to the remaining card. The original formulation of the model accomplished this by assigning an effectively infinite distance to the missing card, but this
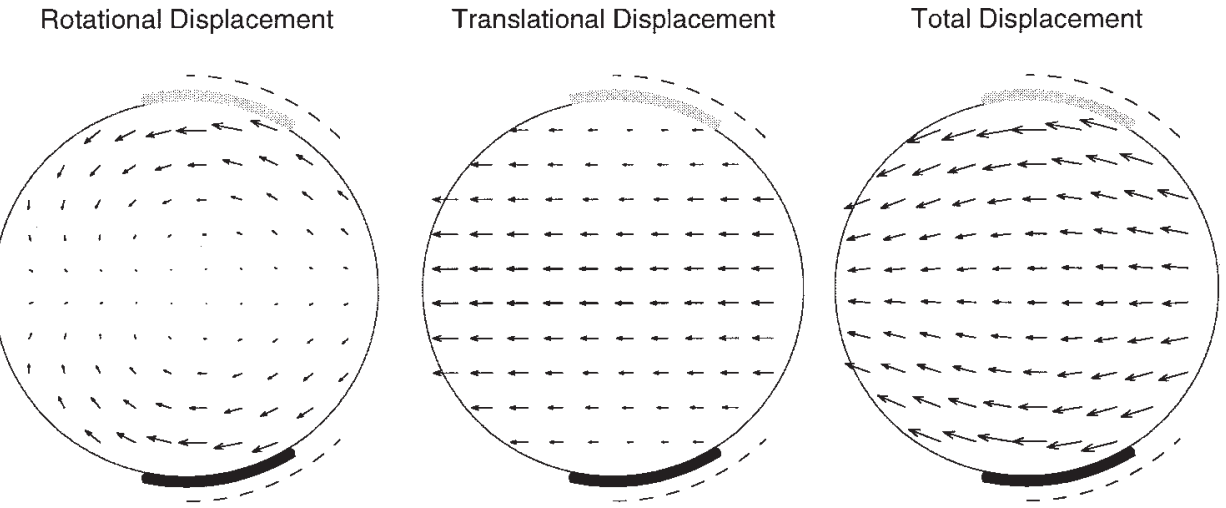

FIGURE 3. Calculated displacement vectors when cards are rotated apart by $25^{\circ}$ : rotational displacement $D_{\text {rot }}$ from eq. (3), translational displacement $D_{\text {trans }}$ from eq. (4), and total displacement $\mathrm{D}_{\text {tot }}$ from eq. (5). 
is unnecessary in our revised scheme. Defining the reference point to be in "standard position" whenever a card is missing gives $\alpha=$ 0 for eq. (2). The values for $d_{w}$ and $d_{b}$ in eq. (4) are thus unimportant, because $\mathrm{W}_{\mathrm{c}}$ and $\mathrm{B}_{\mathrm{c}}$ are null vectors. The model is already insensitive to rotations of the cards and cylinder relative to the room frame, so setting the reference point as if the missing cue card were in its standard position relative to the remaining card poses no problem.

A rat could implement this operation without explicitly calculating a "reference point" by using the one visible cue card to reset its head direction (HD) system. Skaggs et al. (1995) describe an attractor-based model of the HD system, in which feature detectors tuned to egocentric bearings of individual landmarks develop projections to selected HD units as the animal explores the environment. Once these projections are established, they keep the system aligned with the environment. The nature of these attractor networks is such that when two cards are present and have been moved together or apart by a modest amount, so that they provide somewhat conflicting head direction cues, the network will average their influence. When only a single card is present, it can control the alignment of the HD system.

\section{MAXIMUM LIKELIHOOD MODEL}

Our first alternative to the purely descriptive approach of the vector field equations (3)-(5) is to look for a theoretically justified account of map deformation. Let us assume the rat uses a probabilistic method to determine its position, with each landmark an independent source of position information. In this framework, each landmark observation generates a probability distribution for the animal's current location. When the cue cards are in the standard configuration, all evidence sources should agree (in the absence of noise.) But when the cards move closer together or farther apart, the evidence becomes inconsistent. A reasonable response to this situation is to take all the evidence into account and choose the peak of the combined probability distribution as the animal's most likely location. This maximum likelihood approach uses the estimate of position to predict the movement of place field centers, on the assumption that place cell activity indicates where the rat thinks it is. The approach thus offers a probability-theoretic justification for why the firing field map distorts with cue card movement.

Let the probability distribution generated by a landmark observation be a Gaussian function of distance. If the rat perceives its distance to landmark $i$ to be $v_{i}$, the probability that its actual distance to the landmark is $\mathrm{d}_{\mathrm{i}}$ is distributed across a Gaussian annulus (in unobstructed space; the portion within the cylinder forms an arc) with a peak at distance $v_{i}$ and a variance $\sigma^{2}$, which we assume is proportional to $v_{i}^{2}$ :

$$
\mathrm{p}\left(\mathrm{d}_{\mathrm{i}} \mid \mathrm{v}_{\mathrm{i}}\right)=\frac{1}{\mathrm{~A}_{\mathrm{i}}} \exp \left[\frac{-\left(\mathrm{d}_{\mathrm{i}}-\mathrm{v}_{\mathrm{i}}\right)^{2}}{\mathrm{v}_{\mathrm{i}}^{2}}\right]
$$

where $A_{i}$ is the area under the annulus. Making $\sigma$ proportional to the perceived distance to the landmark causes distributions to scale in accordance with Weber's law. Weber's law, a fundamental precept of psychophysics, states that the magnitude of the just noticeable difference in a stimulus is proportional to the stimulus intensity. Scaling the width of each Gaussian based on distance from the controlling landmark was also used in the place field model of O'Keefe and Burgess (1996).

Within the cylinder, the set of locations at distance $d_{i}$ from landmark i forms an arc centered on the landmark. A single landmark is therefore insufficient for a unique determination of location, but a pair of landmarks is usually adequate. We assume that both edges of each cue card are used as landmarks, so four landmarks are normally visible. When one card is deleted, two landmarks are still available.

If landmarks are independent evidence sources, then they can be combined by multiplying their probability distributions. The two cue cards would be truly independent if cards could overlap. Even when this is prohibited, the cards are nearly independent. But the four cue card edges are not independent, since if we know the location of the left edge of a card, the right edge can only be in one location, determined by the card's fixed width. Of course, the rat might not assume that the width is fixed. We will treat the landmarks as independent for purposes of evidence combination, as a naive observer, unaware that a card's two edges should move in unison, might do. For our maximum likelihood estimator, we therefore approximate the joint probability distribution as:

$$
\mathrm{p}\left(\mathrm{x}, \mathrm{y} \mid \mathrm{v}_{1}, \ldots, \mathrm{v}_{4}\right)=\prod_{\mathrm{i}=1}^{4} \mathrm{p}\left(\mathrm{x}, \mathrm{y} \mid \mathrm{v}_{\mathrm{i}}\right)
$$

The product of several Gaussian arcs that intersect at a single point is, roughly, a Gaussian bump. Thus, the Gaussian firing rate distribution of a place cell whose field center is at distances $v_{1}$ through $v_{4}$ from the four landmarks when the cards are in standard position can be viewed as an estimate of $\mathrm{p}(\mathrm{x}, \mathrm{y})$. Moving the rat (changing $\mathrm{x}$ and $\mathrm{y}$ ) samples the cell's firing rate distribution at different arena locations. Moving the cue cards alters the observed combinations of $\mathrm{v}_{\mathrm{i}}$ 's and thus changes the entire distribution.

The joint probability distribution $\mathrm{p}\left(\mathrm{x}, \mathrm{y} \mid \mathrm{v}_{1}, \cdots, \mathrm{v}_{4}\right)$ need not be computed directly. It suffices to calculate $\mathscr{L}_{\mathrm{xy}}$, the log likelihood, which has the advantage of eliminating the exponential functions and replacing a product with a sum:

$$
\begin{aligned}
\mathscr{L}_{\mathrm{xy}}=\log \mathrm{p}\left(\mathrm{x}, \mathrm{y} \mid \mathrm{v}_{1}, \ldots, \mathrm{v}_{4}\right)=\log \prod_{\mathrm{i}=1}^{4} \mathrm{p}\left(\mathrm{x}, \mathrm{y} \mid \mathrm{v}_{\mathrm{i}}\right) \\
=\sum_{\mathrm{i}=1}^{4} \log \mathrm{p}\left(\mathrm{x}, \mathrm{y} \mid \mathrm{v}_{\mathrm{i}}\right)=-\sum_{\mathrm{i}=1}^{4}\left(\log \mathrm{A}_{\mathrm{i}}-\frac{\left(\mathrm{d}_{\mathrm{i}}^{\mathrm{xy}}-\mathrm{v}_{\mathrm{i}}\right)^{2}}{\mathrm{v}_{\mathrm{i}}^{2}}\right)
\end{aligned}
$$

The maximum of the log likelihood will be found at the same location as that of the underlying probability distribution. To find the location $\left[x^{*}, y^{*}\right]$ of the peak of the probability distribution, we eliminate constant terms and calculate: 


$$
\left[x^{*}, y^{*}\right]=\underset{x, y}{\operatorname{argmax}} \sum_{i=1}^{4} \frac{-\left(d_{i}^{x y}-v_{i}\right)^{2}}{v_{i}^{2}}
$$

An interesting consequence of using Weber's law scaling is that nearer landmarks have greater influence on the location of the peak. To see this, consider the one-dimensional case with landmarks at location $l_{1}$ and $l_{2}$ on the real line. At any point $x$, the distances to these landmarks are $\mathrm{d}_{1}^{\mathrm{x}}=\mathrm{x}-\mathrm{l}_{1}$ and $\mathrm{d}_{2}^{\mathrm{x}}=\mathrm{x}-\mathrm{l}_{2}$. Suppose we move the landmarks and try to estimate our position based on the observed distances $\mathrm{v}_{1}$ and $\mathrm{v}_{2}$, using $\log$ likelihood:

$$
\mathscr{L}_{\mathrm{x}}=-\left(\mathrm{d}_{1}^{\mathrm{x}}-\mathrm{v}_{1}\right)^{2} / \mathrm{v}_{1}^{2}-\left(\mathrm{d}_{2}^{\mathrm{x}}-\mathrm{v}_{2}\right)^{2} / \mathrm{v}_{2}^{2}
$$

The local maximum, attained when $\mathrm{d} \mathscr{L}_{\mathrm{x}} / \mathrm{dx}=0$, is

$$
\mathrm{x}^{*}=\frac{\left(\mathrm{l}_{1}+\mathrm{v}_{1}\right) \mathrm{v}_{2}^{2}+\left(\mathrm{l}_{2}+\mathrm{v}_{2}\right) \mathrm{v}_{1}^{2}}{\mathrm{v}_{1}^{2}+\mathrm{v}_{2}^{2}}
$$

which bears a strong resemblance to eq. (3), substituting $l_{1}+v_{1}$ for $\mathrm{W}_{\mathrm{x}, \mathrm{y}}, \mathrm{v}_{1}^{2}$ for $\mathrm{d}_{\mathrm{w}}, \mathrm{l}_{2}+\mathrm{v}_{2}$ for $\mathrm{B}_{\mathrm{x}, \mathrm{y}}$, and $\mathrm{v}_{2}^{2}$ for $\mathrm{d}_{\mathrm{b}}$. Equation (3) was chosen by Fenton et al. to give greater weight to nearby landmarks.

Unlike the vector field model, the maximum likelihood model operates in room coordinates and makes no assumptions about head direction reset. When the two cards rotate by equal amounts, all points rotate around the center by a corresponding amount. When a card is removed, we simply omit the corresponding terms from the sum; there is no inconsistency among the remaining cues so the fields rotate without distortion. But when the cards move closer together or farther apart, the four Gaussian arcs defined by the individual landmarks shift relative to each other, and the map undergoes stretching and shrinking.

We implemented this model with a grid of points spaced $1 \mathrm{~cm}$ apart. The 76-cm-diameter cylinder contained 4,513 of these points. To plot the vector field with the cards rotated, we calculated $\mathrm{v}_{\mathrm{i}}{ }^{\prime}$ values for the rotated landmarks viewed from all points on the grid. We then selected an evenly spaced subset of points from the interior of the cylinder, and for each point $[\mathrm{x}, \mathrm{y}]$ representing a firing field center, we found $\left[\mathrm{x}^{*}, \mathrm{y}^{*}\right]$, the peak of $\mathrm{p}\left(\mathrm{x}, \mathrm{y} \mid \mathrm{v}_{1}{ }^{\prime}, \cdots, \mathrm{v}_{4}{ }^{\prime}\right)$. This was the grid location of the cell's firing field center with the cards rotated. We then drew a vector from $[x, y]$ to $\left[x^{*}, y^{*}\right]$.

As shown in Figure 4a, the results of this formulation do not always match that of the Fenton et al. (2000b) model. Specifically, when the two cards move closer together by $25^{\circ}$, most of the vectors point roughly east, but there is a region around the arena center where the vectors are noticeably attenuated. This is because, at the center of the arena, the distance to all four landmarks is equal to the arena radius, and this distance is unchanged by cue card rotation. Another striking feature of the plot is the sharp discontinuity in the eastern portion of the arena, around the vertical line joining the left edge of the white card to the right edge of the black card. The vectors suddenly switch direction in this region. This is the result of eq. (9) choosing a single location for the maximum value of $\mathscr{L}_{\mathrm{xy}}$. Points on the line actually have two maxima, one on either side. As one moves off the line in either direction, the symmetry is broken. These unexpected results are a consequence of the geometry of the arena (a concave, symmetric interior with land- marks distributed asymmetrically around the edges) and our assumption that distance from landmarks is the rat's sole source of evidence for its position.

A plausible alternative hypothesis is that rats determine their position based on angles between pairs of landmarks, i.e., the difference in their relative bearings, or equivalently, the retinal angle subtended by a line connecting them. We explored this possibility in a second model. With four visible landmarks there are six possible landmark pairs but, for simplicity, we used just the four pairs of circularly adjacent landmarks, since the additional pairs would only provide redundant information. (Only three angles are actually independent; we use four to maintain symmetry in the model.) Note that the retinal angle between a card's left and right edge decreases with distance from the card, while the retinal angle between edges of the white card and the black card is a function of both distances to the cards and the card separation angle.

Let $\mathrm{u}_{\mathrm{ij}}$ be the rat's perceived angle between landmarks $\mathrm{i}$ and $\mathrm{j}$, and let $\mathrm{a}_{\mathrm{ij}}^{\mathrm{xy}}$ be the actual bearing difference between these landmarks viewed from location $[\mathrm{x}, \mathrm{y}]$ with the cards in standard position. We can define a Gaussian probability distribution for position based on the perceived angle between a pair of distinct landmarks as:

$$
\mathrm{p}\left(\mathrm{x}, \mathrm{y} \mid \mathrm{u}_{\mathrm{ij}}\right)=\frac{1}{\mathrm{~K}} \exp \left[\frac{-\left(\mathrm{a}_{\mathrm{ij}}^{\mathrm{xy}}-\mathrm{u}_{\mathrm{ij}}\right)^{2}}{2 \sigma_{\mathrm{a}}^{2}}\right]
$$

where $K=\sqrt{2 \pi \sigma_{\mathrm{a}}^{2}}$ is a normalization constant, and the variance $\sigma_{\mathrm{a}}^{2}$ may either be fixed, or proportional to $\mathrm{u}_{\mathrm{ij}}$. Note: for circular values such as bearings, a circular distribution should be used in place of the normal distribution. But here our feature detectors are really measuring the lengths of the arcs between pairs of landmarks. Although these values are bounded by the circumference of the arena, they are not periodic: an arc subtending a little more than $0^{\circ}$ of the visual field is not similar to an arc subtending almost $360^{\circ}$. So a normal distribution is used.

Once again, we make the simplifying assumption that evidence sources are independent, so that the overall probability distribution is the product of the probability functions for the four landmark pairs $\mathrm{u}_{12}, \mathrm{u}_{23}, \mathrm{u}_{34}$, and $\mathrm{u}_{41}$. The result (Fig. $4 \mathrm{~b}$ ) shows good behavior in the vicinity of the cue cards but anomalous results in other parts of the cylinder. This plot was made with $\sigma_{\mathrm{a}}$ proportional to $\mathrm{u}_{\mathrm{ij}}$, using a constant value produced slightly less satisfactory results. In either case, at locations far from either cue card the angles between landmarks change fairly slowly with position, producing a shallow gradient with broad peaks. When the two cards move in a nonrigid fashion, the location of a firing field peak in the western half of the cylinder can shift by a large amount even though the change in magnitude of the probability values is small. Therefore, angle-based features are also not ideal for determining position in the cylinder.

The single landmark feature that produced the best firing field deformation pattern was allocentric bearing, i.e., bearing to the landmark with respect to some external coordinate system independent of the present heading. The result is shown in Figure 4c. 

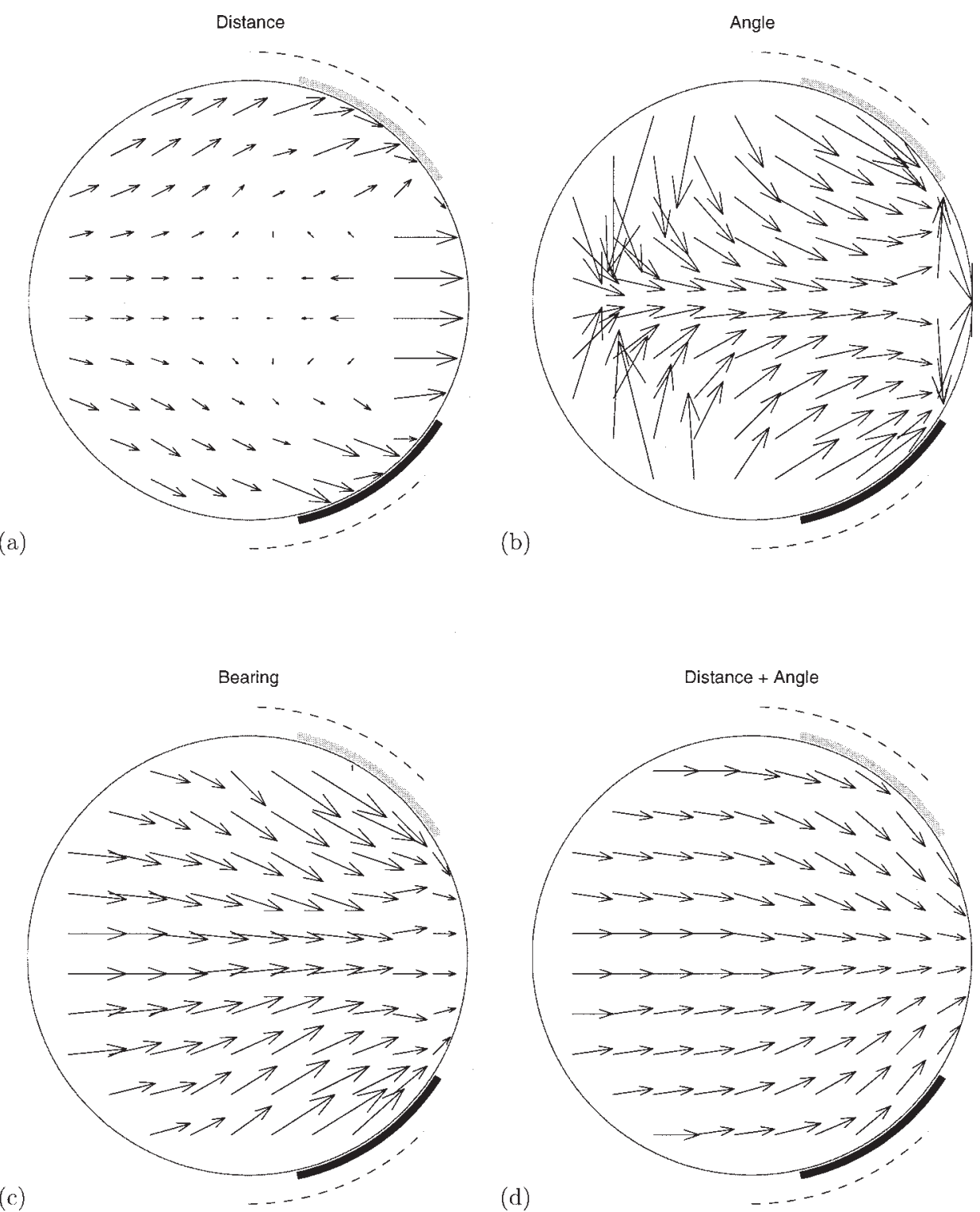

FIGURE 4. Maximum likelihood estimation of firing field movement when the cards are rotated closer together by $25^{\circ}$, using (a) distance to landmarks, (b) angles between pairs of landmarks, (c) allocentric bearings to landmarks, or (d) a combination of distance and angle information.

Neurons in the rodent head direction system have been shown to encode the animal's heading with respect to the environment (Taube et al., 1990b). This suggests that rats are capable of computing allocentric bearings of landmarks. Behavioral experiments in gerbils also indicate that rodents can use allocentric bearing information to disambiguate landmarks (Collett et al., 1986). Furthermore, in a familiar environment the alignment of the rodent head direction system is known to be controlled by visual landmarks (Taube et al., 1990a). Therefore, to compute allocentric bearings in our maximum likelihood model, we assume that the animal's heading reference (east) is defined as midway between the white and black cue cards, just as in our vector field model. Moving the cards together or apart by equal but opposite amounts leaves the reference point unchanged, and thus does not affect the alignment of the head direction system, although individual landmark bearings will of course shift. Rotating the cards by identical amounts rotates the heading reference as well, so the rat does not notice any bearing change in that situation either.

In the standard cue configuration, the reference point is half a card width clockwise from the right edge of the white card, and half a card width counterclockwise from the left edge of the black card. When one card is deleted, we assume that "east" is in its standard position relative to the remaining card. Hence, with one card present, firing field centers can rotate but will not deform.

The overall best result was obtained by combining distance with either the angle or bearing based features. The distance-based proba- 

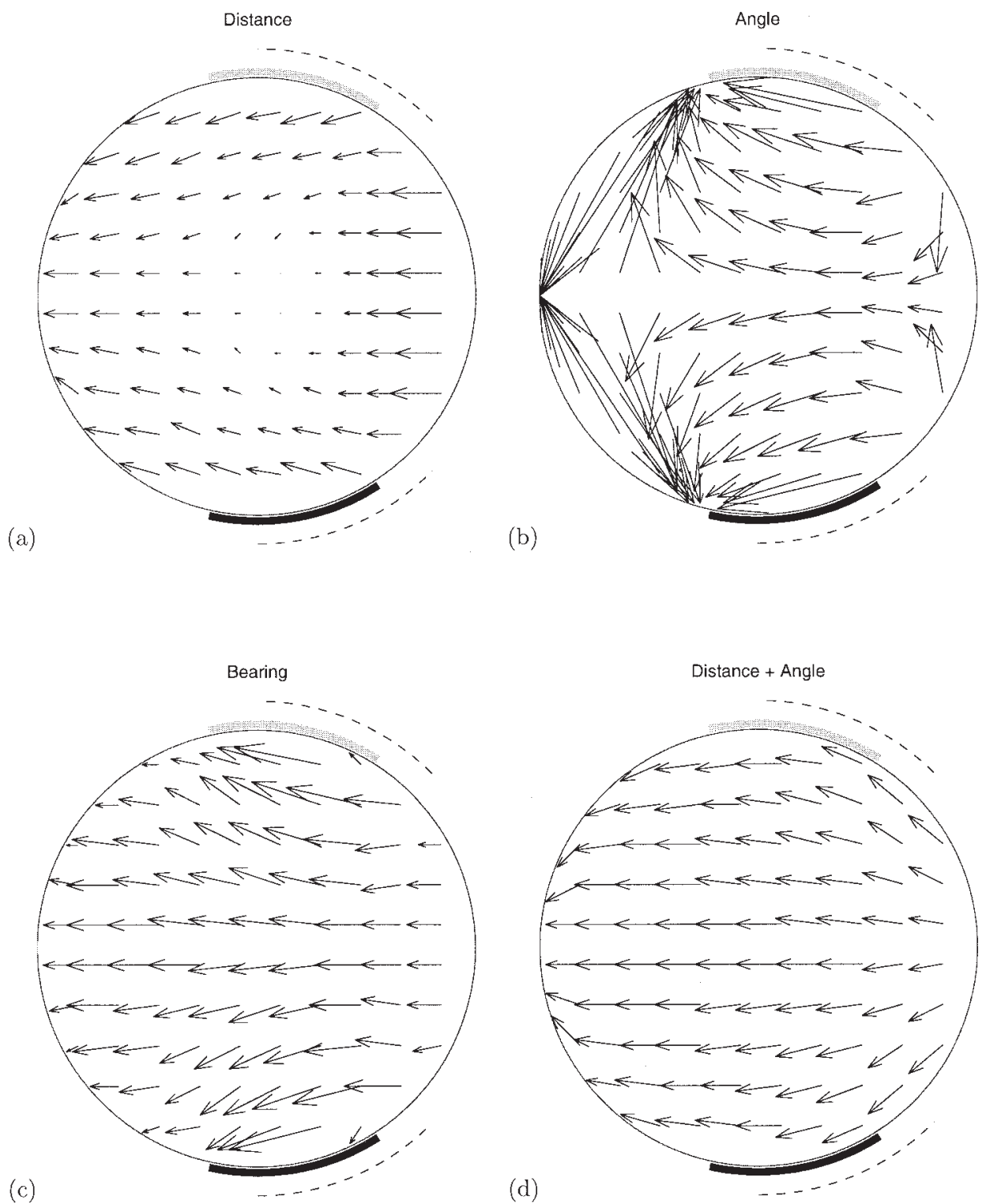

FIGURE 5. Maximum likelihood estimation of firing field movement when the cards are rotated apart by $25^{\circ}$, using (a) distance to landmarks, (b) angles between pairs of landmarks, (c) allocentric bearings to landmarks, or (d) a combination of distance and angle information.

bility function (Fig. 4a) has a stronger gradient in the western half of the cylinder than the angle-based function (Fig. $4 \mathrm{~b})$. Combining the log likelihoods by simple addition allows each to compensate for the other's shortcomings. As shown in Figure 4d, the resulting vector fields are very close in appearance to those of Fenton et al. We set $\sigma_{\mathrm{a}}$ to unity in this case. The locations of peak firing were estimated by:

$$
\left[\mathrm{x}^{*}, \mathrm{y}^{*}\right]=\underset{\mathrm{x}, \mathrm{y}}{\operatorname{argmin}}\left[\sum_{\mathrm{i}=1}^{4} \frac{\left(\mathrm{d}_{\mathrm{i}}^{\mathrm{xy}}-\mathrm{v}_{\mathrm{i}}\right)^{2}}{\mathrm{v}_{\mathrm{i}}^{2}}+\sum_{\mathrm{i}, \mathrm{j}}\left(\mathrm{a}_{\mathrm{ij}}^{\mathrm{xy}}-\mathrm{u}_{\mathrm{ij}}\right)^{2}\right]
$$

Similar results were obtained using distance plus allocentric bearing.

Figure 5 shows the output of various versions of the maximum likelihood model when the cards are moved apart by $25^{\circ}$. Once again, a combination of distance and angle features produces the best results. Using this combination, the mean horizontal displacement of firing fields was $+6.56 \mathrm{~cm}$ when the cards were moved together, versus $-7.32 \mathrm{~cm}$ when they were moved apart. These values are somewhat larger than the predictions reported by Fenton et al. (2000b): $+5.59 \mathrm{~cm}$ for cards together and $-5.95 \mathrm{~cm}$ for cards apart. They are also larger than our own numerical simulations of eq. (5) using a grid with $1 \mathrm{~cm}$ resolution $(+4.71 \mathrm{~cm}$ together, $-5.09 \mathrm{~cm}$ apart). But in all three simulations, the magnitude of the displacement in the cards-together case is approximately $90 \%$ of that in the cards-apart case. In the maximum likelihood model, the larger displacement values can be attributed to 
the particular choice of feature values selected (angle plus distance) and their relative weightings.

\section{ATTRACTOR BUMPS}

Dynamic systems, or "attractor bump" networks, are a popular approach to modeling aspects of hippocampal place cells (Samsonovich and McNaughton, 1997), and have been widely adopted by hippocampal modelers (Redish and Touretzky, 1998; Redish, 1999; Doboli et al., 2000; Káli and Dayan, 2000). One-dimensional attractor models have been used to model the head direction system (Zhang, 1996; Redish et al., 1996; Goodridge and Touretzky, 2000), orientation tuning in visual cortex (Ben-Yishai et al., 1997), and the oculomotor system (Seung, 1996). Twodimensional (2D) attractor networks have been proposed as models of hippocampus, superior colliculus (Droulez and Berthoz, 1991; Pouget et al., 2002), and motor cortex (Lukashin et al., 1996). Here we examine the ability of an attractor network to function as a deformable map, producing the stretching and shrinking effects observed in the Fenton and Muller two-card experiment.

We begin with a population of place cells arranged as a $2 \mathrm{D}$ grid. Let each cell have strong excitatory connections to the cells nearby, weaker excitatory connections to cells somewhat further away, and inhibitory connections to all the remaining cells. With appropriate parameter settings, a network organized this way will have an infinite number of stable states, each consisting of a "bump" of activity localized to some region of the grid. Such a state is analogous to the population activity observed in the hippocampus, because when the rat is at a particular location, the place cell whose field is centered closest to the rat's location will be firing at its maximum rate, while cells whose firing fields just overlap with the rat's location will fire at lesser rates, and cells whose fields are far from the rat's location will be quiescent. As the rat moves through the environment, the activity pattern over the place cell population shifts to reflect this.

If the units comprising an attractor network are initialized with random activity levels, the network will settle into a stable state with a well-formed bump at a random location. However, if a smoothly varying external input is applied to some region of the grid, the bump will tend to form in the region of maximal external input. The attractor network can thus be regarded as a parallel, distributed mechanism for finding the peak of an input signal projected onto the grid.

To produce visual control of firing fields, the external input may be taken from a collection of visual feature detectors tuned to landmark distances, or bearings, or both. To model the two-card experiment, we created a separate set of feature detectors for each landmark. For the ith landmark, there was a set of distance detectors $F_{i, j}$ tuned to various distances $r_{j}$, and a set of bearing detectors $\mathrm{G}_{\mathrm{i}, \mathrm{j}}$ tuned to various allocentric bearings $\phi_{\mathrm{j}}$. The place cell with firing field centered at $[x, y]$ received an excitatory connection from feature detector $\mathrm{F}_{\mathrm{i}, \mathrm{j}}$ or $\mathrm{G}_{\mathrm{i}, \mathrm{j}}$ if the distance from $[\mathrm{x}, \mathrm{y}]$ to the ith landmark was approximately $r_{j}$, or the allocentric bearing of landmark i viewed from $[\mathrm{x}, \mathrm{y}]$ was approximately $\phi_{\mathrm{j}}$.

Once the feature detectors have been wired up to the place cells, consider the rat entering the environment at the start of a trial. Let the rat's perceived distance from its present location to the ith landmark be $v_{i}$. Those distance detectors $F_{i, j}$ whose preferred distance value is close to $\mathrm{v}_{\mathrm{i}}$ will be active and will supply excitation to the appropriate subset of place cells. A similar situation holds for bearing detectors $\mathrm{G}_{\mathrm{i}, \mathrm{j}}$. A bump of activity will then form over the place cells with its peak centered at roughly the location receiving the greatest amount of feature detector input. The simulated rat has thereby estimated its position in the arena.

The estimate would be exact if the external input to the place cell grid were a circularly symmetric gaussian bump. However, the projections from individual feature detectors to place cells form arcs, not bumps. For example, a distance-based feature detector $F_{i, j}$ will project to the arc of cells centered at distance $r_{j}$ from landmark i. If several arcs cross at a single point at roughly equal angles, the resulting pattern of external input will look bump-like. But this condition does not always hold. Angle-based feature detectors produce very broad arcs in the western half of the arena, and the geometry of the arena and cue cards constrains all arcs to be nearly coincident in that region. Bearing-based feature detectors were used instead because they do not suffer this problem. Another problem is that in the eastern half of the arena, when the cards are moved, distance arcs that once overlapped now merely pass close by, producing elongated patterns of external input that are far from bump-like. These effects were not a problem for the maximum likelihood model because it only looked at the peak of the input distribution; the overall shape of the input was ignored. But the attractor network is sensitive to this shape, and thus requires some refinement of its input features to assure that the peak of the external input is close to the center of the input distribution. We therefore added a feedforward inhibition term $\mathrm{I}_{\mathrm{FD}}$ from the feature detectors to the place cells that was strong enough to cancel any individual arc or intersection of a few arcs, but not the intersection of many arcs. This "decluttered" the external input signal, producing a stimulus that was more focused and bump-like. The amount of this feedforward inhibition was calculated iteratively, using the procedure in the Appendix. Biologically, it may be considered analogous to excitatory projections onto interneurons that in turn make inhibitory projections onto place cells.

The feedfoward inhibition mechanism is separate from the recurrent inhibition component of the attractor network itself. Recurrent inhibition within the attractor network is important for producing a stable bump of the correct shape. The feedforward inhibition term ensures that the bump forms in the correct place.

Another difficulty with the projection from feature detectors to place cells arises close to the arena walls. The $2 \mathrm{D}$ attractor grid extends beyond the arena boundaries, but since the animal cannot experience the environment beyond the walls, feature detectors were not wired up to place cells lying outside the cylinder. When the simulated animal is at a point along the wall, the attractor bump should be centered on a place cell right at the wall. This cell will excite (and receive excitation from) its nearby neighbors, both 
TABLE 1

Attractor Model Parameters

\begin{tabular}{|c|c|}
\hline Parameter & Value \\
\hline No. of place cells & $2025(45 \times 45)$ \\
\hline No. of landmarks (card edges) & 4 \\
\hline Distance FDs per landmark & 43 \\
\hline Distance values $r_{j}$ & $2 \mathrm{j} \mathrm{cm}, 0 \leq \mathrm{j}<43$ \\
\hline Distance SD $\sigma_{\mathrm{d}}$ & $2 \mathrm{~cm}$ \\
\hline Bearing FDs per landmark & 120 \\
\hline Bearing values $\phi_{\mathrm{j}}$ & $3 \mathrm{j}$ deg., $0 \leq \mathrm{j}<120$ \\
\hline Bearing SD $\sigma_{\mathrm{b}}$ & $6^{\circ}$ \\
\hline Feature detector projection $\mathrm{w}_{\mathrm{ik}}^{\mathrm{EF}}$ & $\begin{array}{l}\text { per eqs. (14) and (15) } \\
\text { and } w_{\mathrm{ik}}^{\mathrm{EF}}=\mathrm{FD}_{\mathrm{k}}^{\mathrm{xi}, \mathrm{yi}}\end{array}$ \\
\hline Feedforward inhibition $\mathrm{I}_{\mathrm{FD}}$ & per App. A \\
\hline Recurrent excitation $w_{i j}^{\mathrm{EE}}$ & per eq. (22) \\
\hline Recurrent excitation scale factor $k_{\mathrm{EE}}$ & 0.1125 \\
\hline Recurrent excitation SD $\sigma_{\mathrm{EE}}$ & 3.1818 \\
\hline Recurrent inhibition $\mathrm{w}^{\mathrm{EI}}$ & -0.35 \\
\hline Inhibitory interneuron drive $\mathrm{w}^{\mathrm{IE}}$ & 0.12 \\
\hline Interneuron self-inhibition $\mathrm{w}^{\mathrm{II}}$ & -1.6 \\
\hline Initial place cell drive $S_{i}(0)$ & $6\left[\sum_{\mathrm{k}} \mathrm{w}_{\mathrm{ik}}^{\mathrm{EF}} \mathrm{FD}_{\mathrm{k}}-\mathrm{I}_{\mathrm{FD}}\right]_{+}$ \\
\hline Initial interneuron drive $\mathrm{SI}(0)$ & 3.5 \\
\hline Time constants $\tau_{\mathrm{E}}, \tau_{\mathrm{I}}$ & $0.0015,0.004$ \\
\hline Time step $\Delta \mathrm{t}$ & 0.001 \\
\hline
\end{tabular}

FD, feature detector; SD, standard deviation.

those closer to the arena center and those further away (hence outside the wall). However, only the cells within the wall receive external input from the feature detectors; the cells outside the wall do not. Thus, there is a danger that the bump may form some distance short of the wall, as that is where the center of mass of the external input lies. To minimize this possibility, we use relatively weak weights from the feature detectors to the place cell grid, so that the attractor dynamics dominate; the feedforward inhibition assures that the location of the peak of the input is more important than its overall shape.

\section{MODEL DETAILS}

Parameter values are given in Table 1 . The activity of the feature detector $F_{i, j}$, tuned to distance $r_{j}$, in response to perceived distance $\mathrm{v}_{\mathrm{i}}$ from the ith landmark, is:

$$
F_{i, j}\left(v_{i}\right)=\exp \left[\frac{-\left(v_{i}-r_{j}\right)^{2}}{2 \sigma_{d}^{2}}\right]
$$

The activity of feature detector $G_{i, j}$, tuned to bearing $\phi_{j}$, in response to perceived bearing $b_{i}$ from the ith landmark, is calculated (using circular subtraction) as:

$$
\mathrm{G}_{\mathrm{i}, \mathrm{j}}\left(\mathrm{b}_{\mathrm{i}}\right)=\exp \left[-\frac{\left[\mathrm{bi}-\phi_{\mathrm{j}}\right]^{2}}{2 \sigma_{\mathrm{b}}^{2}}\right]
$$

There are 43 distance and 120 allocentric bearing feature detectors (FD) per landmark, giving 652 feature detectors total. Let
$\mathrm{FD}_{\mathrm{k}}^{\mathrm{xy}}$ denote the activity of the kth feature detector (of either type F or type $\mathrm{G}$ ) when the simulated rat is at location $[\mathrm{x}, \mathrm{y}]$ with the cue cards in standard position. Let $\mathrm{i}$ be the index of the place cell with firing field centered at $\left[\mathrm{x}_{\mathrm{i}}, \mathrm{y}_{\mathrm{i}}\right]$. The strengths of the connections from feature detectors to the ith place cell, $\mathrm{w}_{\mathrm{ik}}^{\mathrm{EF}}$, are set equal to the feature detector activations when the rat is at the location that is to be the place field's center: $\mathrm{w}_{\mathrm{ik}}^{\mathrm{EF}}=\mathrm{FD}_{\mathrm{k}}^{\mathrm{xi}, y \mathrm{i}}$.

The attractor network was implemented as a $45 \times 45$ grid of cells, toroidally connected to eliminate edge effects. This assures that all cells have the same number of neighbors, so that in the absence of external input, the attractor bump has a uniform shape everywhere on the grid. The arena was defined as a circular region 38 units in diameter, centered on the grid origin. Each grid unit therefore covered a surface of $4 \mathrm{~cm}^{2}$. The attractor bump was roughly 17 units in diameter, so a bump located at one edge of the cylinder would have minimal effect on cells at the opposite edge via wrap-around on the torus.

Each place cell's activation $V_{i}(t)$ was computed as the sum of recurrent excitation from other place cells, a global shunting inhibition term, and the external input received from the feature detectors. Shunting inhibition was used because it improves the stability of the attractor (Káli and Dayan, 2000). The feature detector input included a previously mentioned feedforward inhibition term. $\mathrm{I}_{\mathrm{FD}}$, and was thresholded at zero:

$$
\begin{aligned}
\mathrm{V}_{\mathrm{i}}(\mathrm{t}+\Delta \mathrm{t})=\sum_{j} \mathrm{w}_{\mathrm{ij}}^{\mathrm{EE}} S_{j}(\mathrm{t})+\mathrm{w}^{\mathrm{EI}} \mathrm{SI}(\mathrm{t}) \mathrm{V}_{\mathrm{i}}(\mathrm{t}) \\
+\left[\sum_{\mathrm{k}} \mathrm{w}_{\mathrm{ik}}^{\mathrm{EF}} \mathrm{FD}_{\mathrm{k}}-\mathrm{I}_{\mathrm{FD}}\right]_{+}
\end{aligned}
$$

$V_{i}(t)$ is analogous to the cell's membrane potential. The cell's firing rate $\mathrm{F}_{\mathrm{i}}(\mathrm{t})$ is equal to this value thresholded at zero, denoted $\left[\mathrm{V}_{\mathrm{i}}(\mathrm{t})\right]_{+}$. The integral of firing rate over time is the cell's synaptic drive $S_{i}(t)$, the influence it exerts on other cells, governed by a time constant $\tau_{\mathrm{E}}$ (Pinto et al., 1996):

$$
\begin{gathered}
\mathrm{F}_{\mathrm{i}}(\mathrm{t})=\left[\mathrm{V}_{\mathrm{i}}(\mathrm{t})\right]_{+} \\
\mathrm{S}_{\mathrm{i}}(\mathrm{t}+\Delta \mathrm{t})=\mathrm{S}_{\mathrm{i}}(\mathrm{t})+\left[-\mathrm{S}_{\mathrm{i}}(\mathrm{t})+\mathrm{F}_{\mathrm{i}}(\mathrm{t})\right] \frac{\Delta \mathrm{t}}{\tau_{\mathrm{E}}}
\end{gathered}
$$

A global inhibitory unit receives excitation from all place cells and makes recurrent inhibitory projections back to them and to itself. The equations for this inhibitory unit are:

$$
\begin{gathered}
\mathrm{VI}(\mathrm{t}+\Delta \mathrm{t})=\mathrm{w}^{\mathrm{IE}} \sum_{j} \mathrm{~S}_{\mathrm{j}}(\mathrm{t})+\mathrm{w}^{\mathrm{II}} \mathrm{SI}(\mathrm{t}) \\
\mathrm{FI}(\mathrm{t})=[\mathrm{VI}(\mathrm{t})]_{+} \\
\mathrm{SI}(\mathrm{t}+\Delta \mathrm{t})=\mathrm{SI}(\mathrm{t})+[-\mathrm{SI}(\mathrm{t})+\mathrm{FI}(\mathrm{t})] \frac{\Delta \mathrm{t}}{\tau_{I}}
\end{gathered}
$$

The strengths of the recurrent connections $\mathrm{w}_{\mathrm{ij}}^{\mathrm{EE}}$ between cells $\mathrm{i}$ and $j$ on the torus are a Gaussian function of the distance $d_{i j}$ between them:

$$
\mathrm{w}_{\mathrm{ij}}^{\mathrm{EE}}=\mathrm{k}_{\mathrm{EE}} \exp \left(-\mathrm{d}_{\mathrm{ij}}^{2} / \sigma_{\mathrm{EE}}^{2}\right)
$$



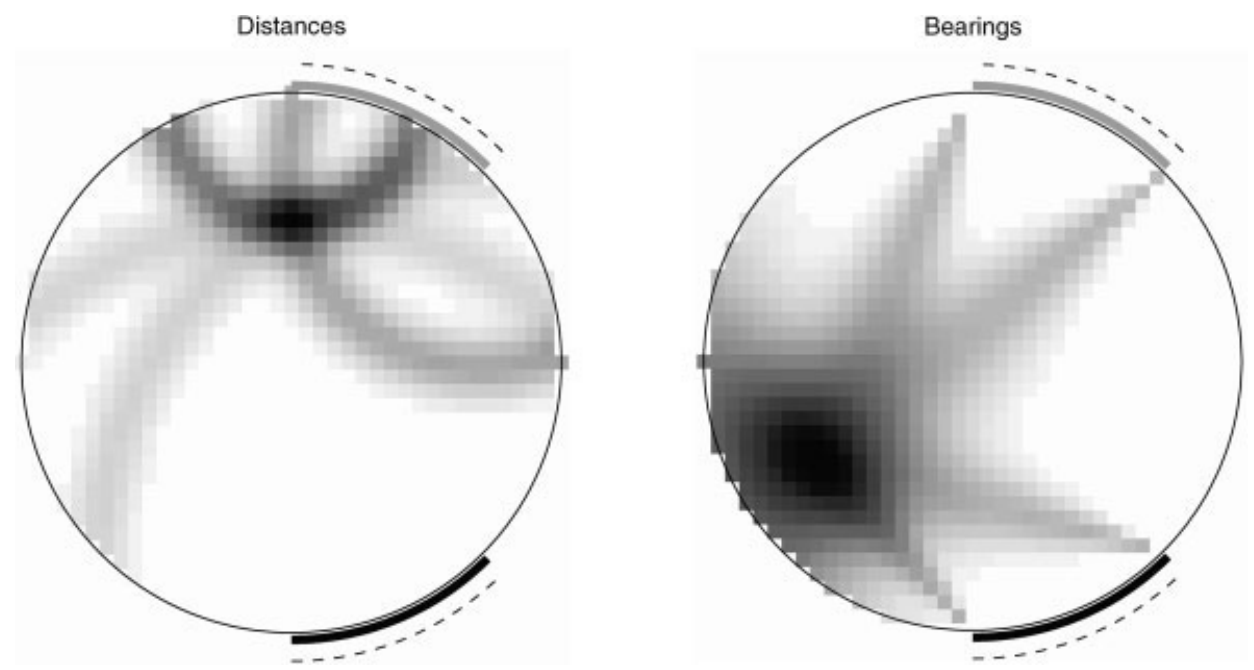

FIGURE 6. Feature detector activity projected onto the place cell grid with the cue cards in standard position. Left: distance-based detectors produce gaussian arcs centered on the four card edges. Right: allocentric bearing-based detectors produce Gaussian cones emanating from the card edges.

For each of the four landmarks there were 43 distance-based Gaussian feature detectors, tuned to even distance values from 0 to $84 \mathrm{~cm} . \sigma_{\mathrm{d}}$ was $2 \mathrm{~cm}$, and the distance feature detectors were weighted relative to each other to place more emphasis on detectors tuned to small distances. The weighting function was $24 /(24+d)$, where $\mathrm{d}$ is the distance (in $\mathrm{cm}$ ) to the landmark, so the relative weights from distance-based feature detectors varied from 1.0 down to 0.22 . In addition, there were 120 allocentric bearing feature detectors tuned to bearing values from $0^{\circ}$ to $357^{\circ}$ in increments of $3^{\circ}$. $\sigma_{\mathrm{b}}$ was $6^{\circ}$, and all the bearing detectors had uniform weights. Figure 6 shows typical activation patterns that distance and bearing-based feature detectors transmit to the place cell grid.

\section{RESULTS FROM THE ATTRACTOR MODEL}

Figures 7 and 8 show the map distortion patterns when the cue cards are moved together or apart by $25^{\circ}$. The results are similar to the vector field and maximum likelihood models, though a little less smooth, due in part to the lower resolution of the grid. The mean horizontal movement of firing fields in this model, measured on a grid of 1129 points, was $+6.70 \mathrm{~cm}$ for the cards together case, and $-6.98 \mathrm{~cm}$ for the cards apart case. These values are comparable to those of the maximum likelihood model and larger than the predictions of the vector field model. The cards-together value is $96 \%$ of the cards-apart value.

The attractor network model also reproduces an independent finding of Fenton et al. (2000a), a reduction in peak firing rates when the cards were rotated together or apart, as shown in Table 2. Peak activation levels in dimensionless units for three cells are shown in Figure 9. The mean peak activation (over all 1,129 cells) declined by $10 \%$ in the cards-apart condition, and by $12 \%$ in the cards-together condition. Fenton et al. report reductions in the mean centroid firing rate, i.e., the firing rate of a cell in the centroid pixel of its firing field, of 36\% (apart) and 35\% (together), and reduction in the mean in-field firing rate of 21\% (apart) and 15\% (together).

The reduction in peak activation in the model is a consequence of the feature detector input becoming defocused when the cards

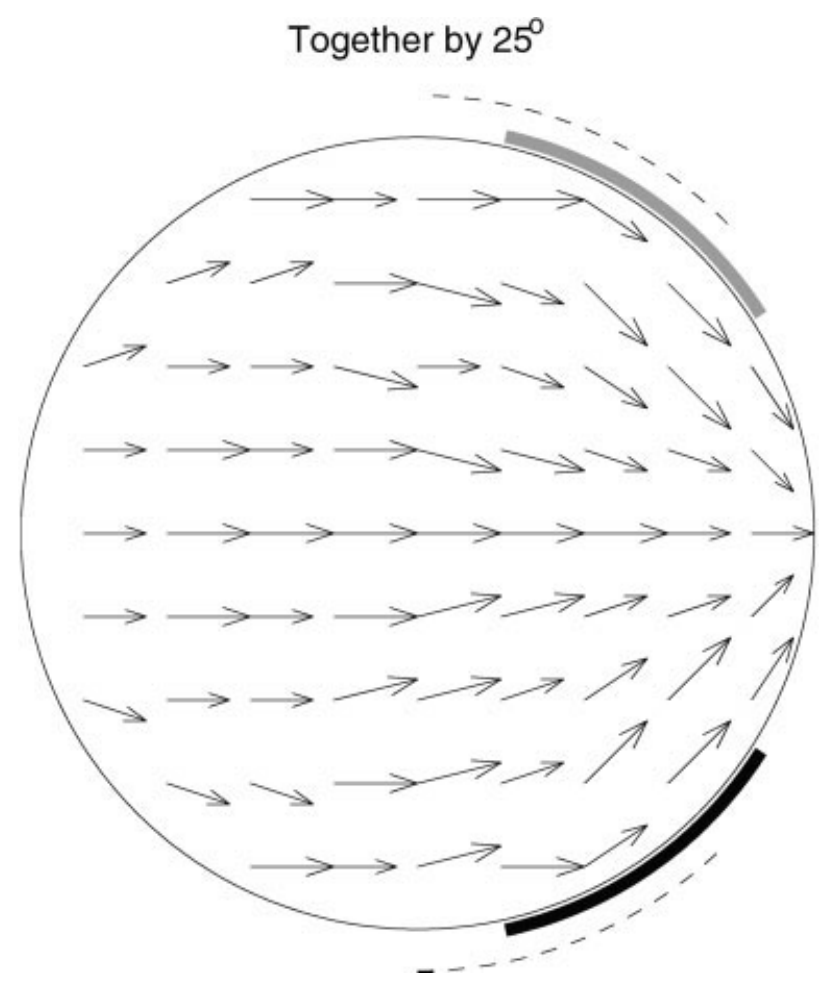

FIGURE 7. Map distortion in the attractor model with the cards rotated closer together by $25^{\circ}$. 


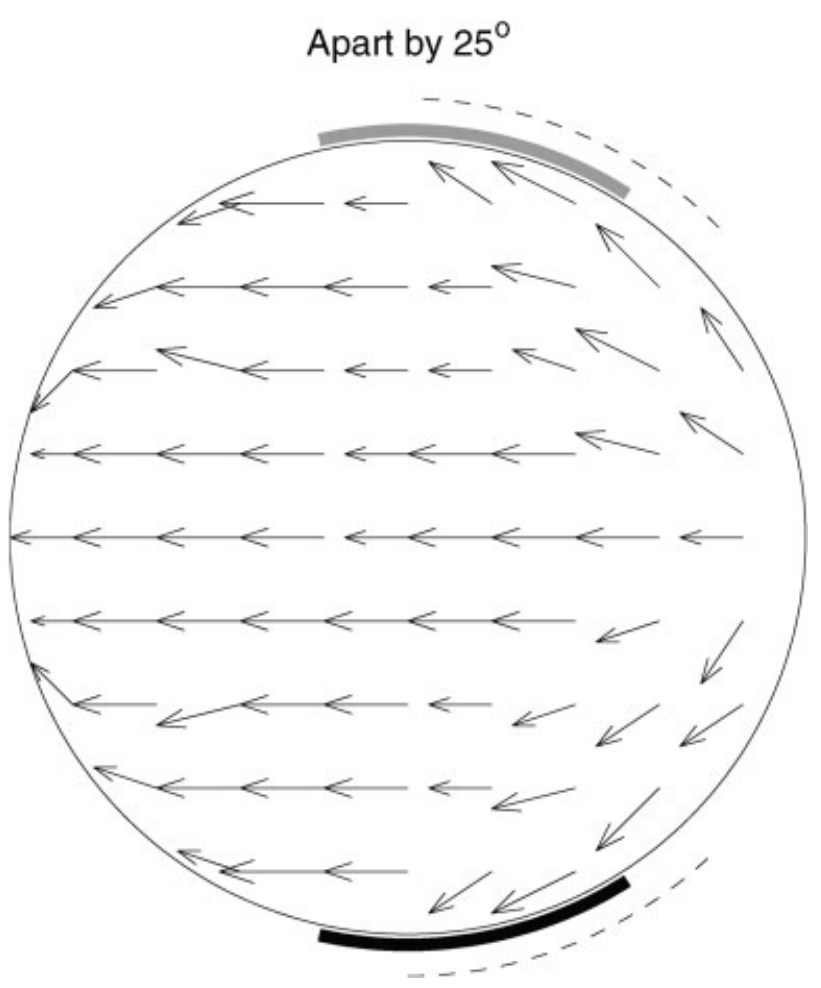

FIGURE 8. Map distortion in the attractor model with the cards rotated farther apart by $25^{\circ} \mathrm{C}$.

are moved out of standard position. The Gaussian arcs from the various feature detectors no longer intersect perfectly at a single point, so place cells at the center of the stimulus bump receive less total input than before.

Figure 9 also shows that the shapes of firing fields distort along the direction of cue card motion. The least distortion is observed at the center of the arena, where only the card bearings change, not the distances.

\section{DISCUSSION}

\section{Comparison of Models}

The vector field model only addresses movement of place field centers. The maximum likelihood model makes similar predictions, but it also predicts the decrease in peak firing rate seen with cue card movement: because sensory inputs are no longer in perfect register, the peak likelihood value is lower. The attractor network model also predicts field movement and reduction in peak firing rate, and furthermore, it generates actual firing fields. The changes in firing field shape predicted to result from card movement sometimes entail substantial deformations (Fig. 9, bottom row). These may differ from what is observed experimentally. However, because many parameters can influence firing field shape, and we have not tuned these parameters to optimize this aspect of the model's behavior, we do not include changes in firing field shapes among the significant predictions of the model presented here.

\section{Comparison of Distance Weightings}

In the vector field model, a cue card's influence in the rotational component [eq. (3)] is weighted inversely by distance in order to achieve the desired map distortion effect (leftmost plots in Figs. 2 and 3). A similar effect is obtained in the maximum likelihood model by giving distance-based landmark features a standard deviation $\sigma_{\mathrm{d}}$ proportional to the perceived distance $\mathrm{v}_{\mathrm{i}}$ [Eq. (6)], on the assumption that such perceptual measurements should obey Weber's law. The scaled variance gives the evidence from a closer landmark a steeper gradient than that from more distant landmarks, hence the position estimate shows greater influence by the closer card.

In the model of Káli and Dayan (2000), entorhinal cortex cells are tuned to both distance and egocentric bearing to walls, and the distance tuning is again sharper for closer walls. It is also sharper for walls behind the animal versus those ahead of it. (Káli and Dayan justify this by assuming that if the animal is headed away from a wall, it has been close to the wall recently, and is thus likely to have a more accurate distance estimate based on path integration.) The models described here use allocentric bearing, not relative bearing, so they do not distinguish landmarks ahead of versus behind the animal.

The boundary vector cells (BVCs) of Hartley et al. (2000) use a response function that is a product of two Gaussians: one tuned to distance and one to allocentric bearing. BVC firing rates are calculated by integrating this response function over all points on the surfaces surrounding the rat. We could instead take the firing rate to be the product of the Gaussian response functions at the specific bearing and distance of one point landmark. Compare this to our maximum likelihood and attractor network models which compute the sum of distance-based and bearing-based tuning functions. The log of a product being equal to the sum of the logs, the two approaches appear similar, and we would expect similar map deformation results.

However, BVCs are not place cells. Hartley et al.'s place cells compute a thresholded linear combination of two or more BVCs,

TABLE 2.

Distribution of Peak Activation Levels (in Dimensionless Units) for All 1,129 Place Cells Within the Arena in the Attractor Network Model, With the Cue Cards in Standard Position or Rotated Apart or Together by $25^{\circ}$

\begin{tabular}{lccc}
\hline Statistic measured & $\begin{array}{c}\text { Standard } \\
\text { configuration }\end{array}$ & $\begin{array}{c}\text { Cards } \\
\text { apart } \\
\text { by } 25^{\circ}\end{array}$ & $\begin{array}{c}\text { Cards } \\
\text { together } \\
\text { by 25 }\end{array}$ \\
\hline Mean peak activation level & 1.5374 & 1.3852 & 1.3517 \\
SD & 0.0889 & 0.0739 & 0.0860 \\
Maximum peak activation & 1.9733 & 1.7993 & 1.7554 \\
Minimum peak activation & 1.3252 & 0.9707 & 0.9430 \\
\hline
\end{tabular}


(a)
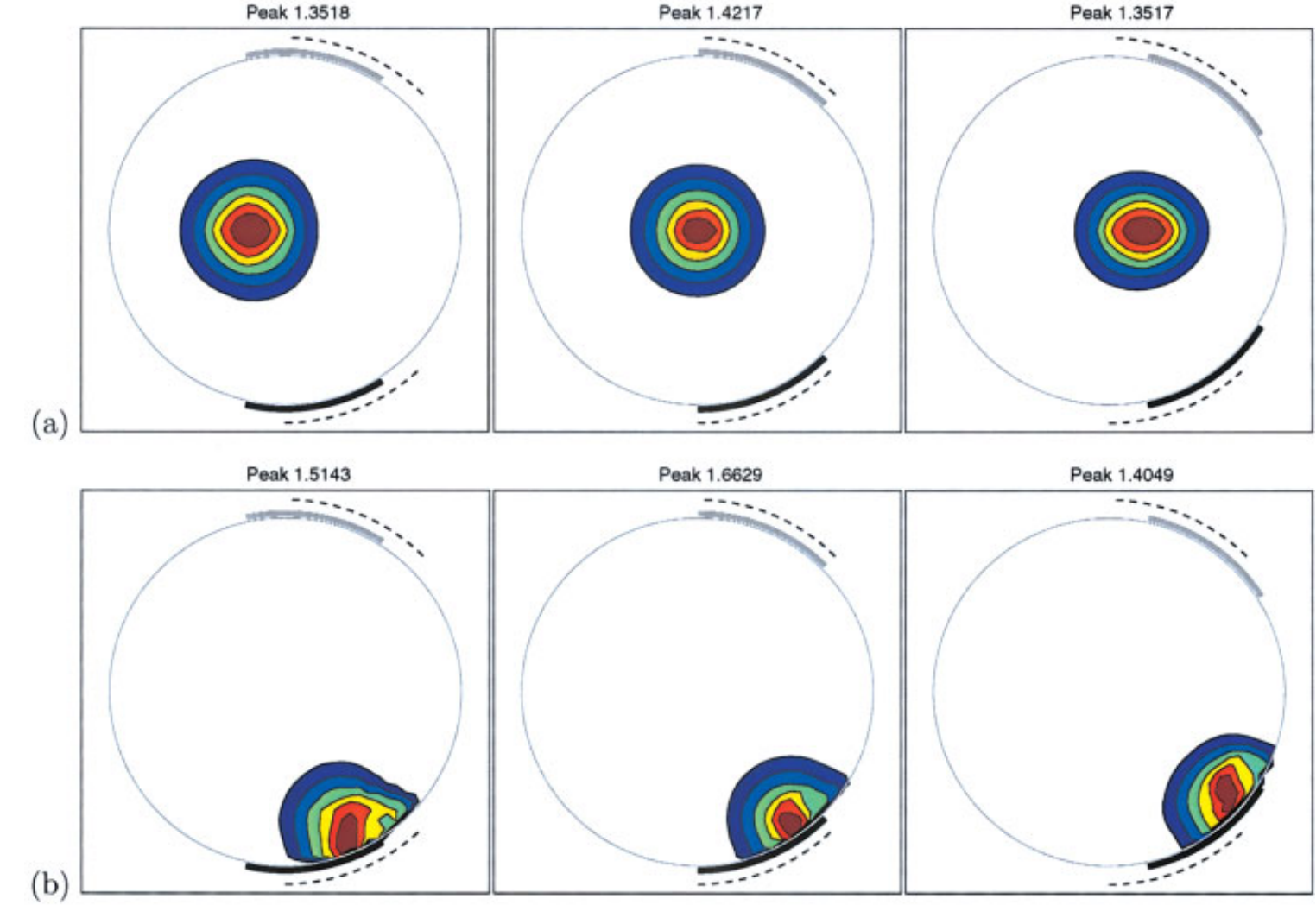

(b)

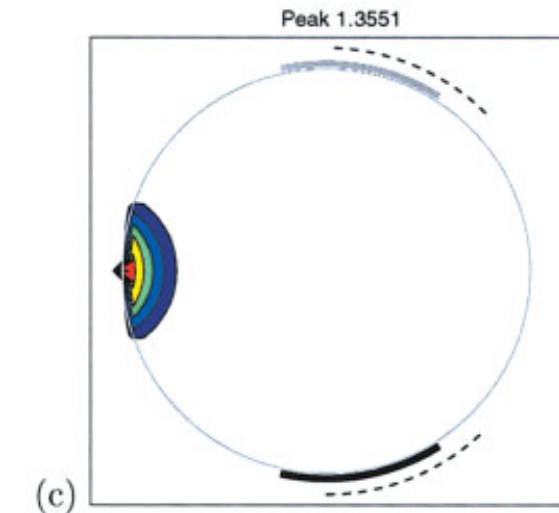

(c)

FIGURE 9. Firing fields of three place cells with cue cards (left) rotated apart by $25^{\circ}$, (center) in standard position, and (right) rotated together by $25^{\circ}$. The top row shows a cell whose field, when the cards are in standard position, is located at the center of the arena. The cell

an extra layer of processing that our model lacks. This appears to be necessary to derive spatially compact place fields when BVC's use entire walls as landmarks. In contrast, our attractor network model uses recurrent connections and network dynamics to produce its roughly Gaussian-shaped firing fields, from inputs with quite varied shapes.

One drawback of using only points as landmarks is that our model does not produce crescent-shaped firing fields along cylinder walls as the Hartley et al. model does. Such fields have been reported by Muller et al. (1987). Their existence suggests that place cells_ at least those associated with boundaries_-should be tightly in the middle row has a field near the black cue card, and the cell in the bottom row has a field at the west edge of the arena. [Color figure can be viewed in the online issue, which is available at www. interscience.wiley.com]

sensory-bound. Place cells not associated with a boundary are presumably driven by distal landmarks and recurrent excitation, making them robust against landmark deletion.

Unlike in our maximum likelihood model, distance-based feature detectors in the attractor network model require a fixed variance, because otherwise, feature detectors tuned to large distance values would supply excitation to broad swaths of place cells, which would deform the shape of the input governing the location of the attractor bump. The maximum likelihood model was unaffected by broad input excitation because it simply picked the single point of maximum activation as the animal's most likely location. But 
the attractor model relies on network dynamics to settle the activity bump over approximately the peak of the input, and is therefore less tolerant of inputs that depart significantly from a compact, roughly symmetric, unimodal shape. Pouget et al. (1998) observed that their recurrent network best approximated a maximum likelihood estimator when the input and output encodings were identical. In our case, that means the afferent projections to place cells should approximate the shape of a stable activity bump. The feedforward inhibition mechanism discussed previously helps to cut off the tails of an elongated input pattern, but is not sufficient in itself to ensure a well-conditioned input. Therefore, in order to reintroduce the effect of Weber's law scaling on a distant landmark's contribution to the activity gradient, we adopted the inverse distance weighting for distance-based feature detectors.

Olypher et al. (2003) recently examined the amount of location information in place cell spikes as rats foraged randomly in the same arena configuration as the one modeled here. They found that local information measures in the center of a place cell's firing field were higher when that field was close to a cue card than when it was far from either card (Olypher et al., 2003). If the feature detectors driving these place cells also carry greater information content when tuned to nearby landmarks, this might provide another justication for weighting nearby features more highly.

\section{Firing Field Deformations}

The strength of the recurrent connections relative to feature detector afferents determines how the attractor network model responds to inconsistent cues. Recurrent connections provide both excitation and inhibition, making firing fields robust against changes in total input and largely preserving their shapes when the cards are rotated. If the recurrent connections are too weak, so that the activity bump with the cards in standard configuration is mainly a result of feature detector input, the reduction in input when a cue card is removed could cause the attractor bump to collapse. And the change in the input distribution when cards are moved together or apart by a significant amount could cause gross distortions in firing field shapes.

In contrast, if recurrent connections are too strong relative to the external input, that input can only influence where on the grid the activity bump appears, and not its shape. But even with a rigid bump shape, the shapes of individual firing fields can still change, since these depend on how the bump moves over the map. In fact, the map distortions visualized in Figures 7 and 8 as shrinking and stretching of the grid of firing field centers necessarily entail some change in firing field shapes. To see this, consider two place cells $\mathrm{i}$ and $\mathrm{j}$ whose firing field centers $\mathrm{c}_{\mathrm{i}}$ and $\mathrm{c}_{\mathrm{j}}$ move closer together, to locations $\mathrm{c}_{\mathrm{i}}{ }^{\prime}$ and $\mathrm{c}_{\mathrm{j}}{ }^{\prime}$, when the cards are rotated together (Fig. 10). Assuming the shape of the activity bump remains rigid due to strong recurrent connections, the firing rate of cell $j$ when the rat is at location $\mathrm{c}_{\mathrm{i}}{ }^{\prime}$ in the rotated environment would be the same as its firing rate when the rat is at $c_{i}$ in the standard environment. But since $c_{i}{ }^{\prime}$ is closer to cell $j$ 's firing field center $c_{j}{ }^{\prime}$, then $c_{i}$ is to $c_{j}$, cell j's firing field has changed.

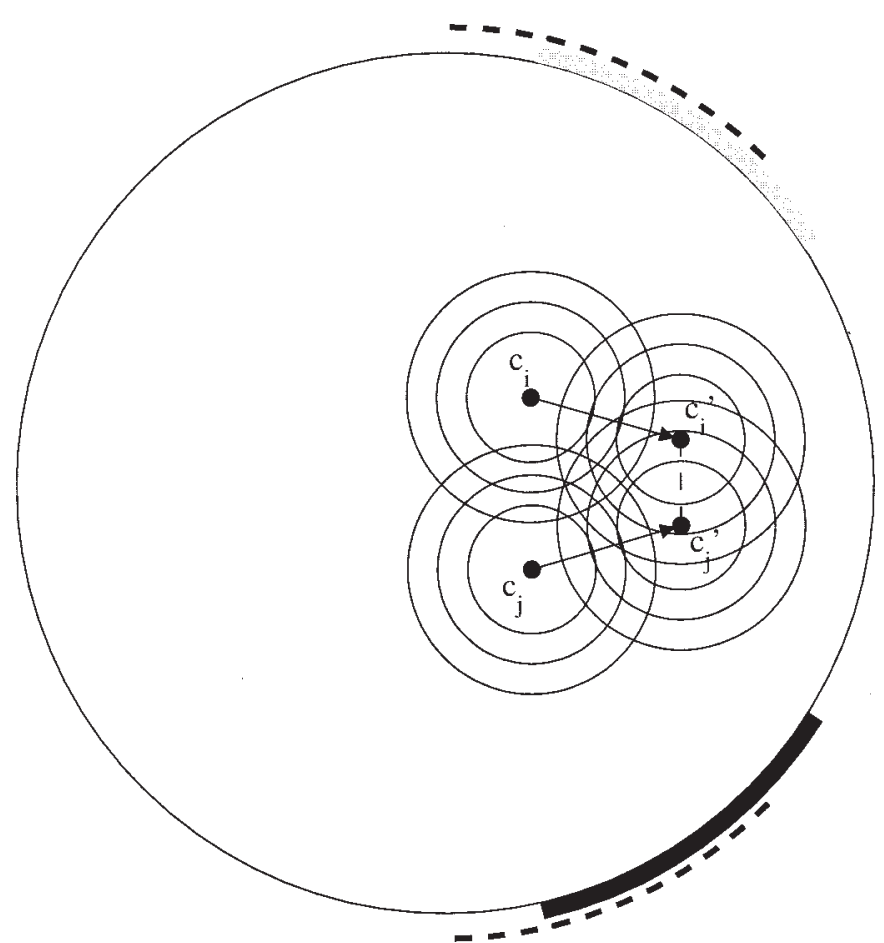

FIGURE 10. Place cells $i$ and $j$ have firing field centers $c_{i}$ and $c_{j}$ that move to $c_{i}{ }^{\prime}$ and $c_{j}{ }^{\prime}$ when the cards are rotated. As a consequence of map distortion, when the rat is at $c_{i}{ }^{\prime}$ with the cards rotated, cell $j$ will be firing at a higher rate than when the rat was at $c_{i}$ with the cards in standard position.

\section{Predictions About Larger Card Movements}

Knierim (2002) argues that recurrent connections must be weak relative to external inputs because in a double cue rotation task where local and distal cues rotated in opposite directions by substantial amounts, some cells followed the local cues, some followed the distal cues, some developed split firing fields, and some remapped entirely.

The cue card manipulations studied here are sufficiently subtle that they do not trigger remapping. Moreover, in our models all cells are influenced by both cue cards; place fields do not dissociate into two sets, one following the white card and one the black card, when the cards move relative to each other. But moving the cards by a greater amount must eventually exceed some threshold beyond which the vector field transformation of Fenton et al. (2000b) no longer applies. Note that rotating the cards apart by $90^{\circ}$ is equivalent to a mirror-image reflection of the arena, where the white and black cards swap places. Unlike in the double cue rotation experiments (Tanila et al., 1997; Knierim, 2002; Brown and Skaggs, 2002), where the discordance is between local cues on a track versus distal cues on the walls, here the discordant cues are of the same type, and presumably equal in salience. We would therefore not expect to see place fields dissociate, as such an effect has never been reported with homogeneous cues. Instead, we predict that at some critical amount of card rotation at $25-90^{\circ}$ either a complete remapping will occur, or one card will lose its influence 
over firing fields, and the fields will rotate with the other card without deforming.

Our current attractor-based model produces neither of these effects. It cannot undergo remapping because there is only one map stored in the recurrent connections. Nor can it reject the influence of one cue card when the inputs from the two cards are so far apart that they do not overlap. A spatially localized stimulus applied far from the flank of an attractor bump should be ignored due to recurrent inhibition (Redish, 1999). However, the feature detectors in our attractor model of the cylinder have broad projections (see Fig. 6), so rotating the cue cards still produces inputs that intersect with and influence the bump location. Thus, for the rat to ignore one card when the separation is increased to $90^{\circ}$, some attentional mechanism not considered in the present work would have to come into play.

\section{Summary}

We have argued that the maximum likelihood formalism gives a satisfying theoretical account of map deformation, in which evidence from multiple independent cues is combined arithmetically to derive a probability distribution for the location of the animal. We assume that the activity of hippocampal place cells reflects this probability distribution. The good agreement between the attractor network and maximum likelihood models supports previous observations that attractor networks can efficiently implement maximum likelihood reasoning (Pouget et al., 1998; Deneve et al., 2001). If rats make near optimal use of available landmarks, they may do so via a network of this sort.

However, the success of both the maximum likelihood and the attractor network models was found to depend on the choice of feature detectors used, since the distribution of landmarks is not symmetric. For the maximum likelihood model the best choice was an equally weighted combination of distance and angle features. For the attractor network model, a combination of distance and allocentric bearing features gave the best performance. These models and the vector field model all predict larger average horizontal displacements of firing field centers in the cards-apart case than in the cards-together case, a natural consequence of the landmark geometry.

The maximum likelihood and attractor network models predict larger horizontal displacements than the vector field model in both the cards-together and cards-apart cases, but this might be remedied by further tweaking of the feature detectors and their relative weightings. The vector field model in turn predicts a significantly larger displacement in the cards-together case $(+3.80 \mathrm{~cm})$ than was observed experimentally by Fenton et al. $(+1.80 \mathrm{~cm})$. None of the models can explain this discrepancy.

Another area in which all the models disagree with the experimental observations is the displacement of fields at the 9 o'clock position, i.e., close to the wall at the western edge of the arena. Fenton et al. report almost no horizontal displacement of firing fields in this region, while the models predict substantial displacements. A potential explanation for this result is that the rat is using distance from the arena wall as another source of location informa- tion. In most locations, this is a less salient cue than the cue cards, but at the 9 o'clock position the cards are maximally distant and the wall is close, so it may receive greater weight and serve to "anchor" the firing field. Note that adding distance from the wall as another type of landmark feature, combined with allocentric bearing information, would allow us to derive crescent-shaped place fields.

Finally, these models raise the question: what is the threshold for cue card motion beyond which the hippocampus either remaps or ignores one of the cards? We are currently investigating a Bayesian model selection account of remapping that may shed light on this question (Fuhs and Touretzky, 2003).

\section{Acknowledgments}

The authors thank two anonymous referees for helpful comments on the manuscript. This work was supported by National Institutes of Health award MH59932 (to D.S.T. and W.E.S.); by National Science Foundation REU supplement to award IIS-9978403 (to D.S.T. for W. W.); by National Science Foundation IGERT training grant DGE-9987588 (to D.S.T. for M.F.); by National Institutes of Health awards NS20686 and NS37150 (to R.U.M.); and by Medical Research Council (UK) (to R.U.M.).

\section{REFERENCES}

Ben-Yishai R, Hansel D, Sompolinsky H. 1997. Traveling waves and the processing of weakly tuned inputs in a cortical network module. J Comput Neurosci 4:57-77.

Brown JE, Skaggs WE. 2002. Concordant and discordant coding of spatial location in populations of hippocampal CA1 pyramidal cells. J Neurophysiol 88:1605-1613.

Collett T, Cartwright BA, Smith BA. 1986. Landmark learning and visuospatial memories in gerbils. J Comp Physiol A 158:835-851.

Deneve S, Latham P, Pouget A. 2001. Efficient computation and cue integration with noisy population codes. Nat Neurosci, 4:826-831.

Doboli S, Minai AA, Best PJ. 2000. Latent attractors: a model for contextdependent place representations in the hippocampus. Neural Comput, 12:1003-1037.

Droulez J, Berthoz A. 1991. A neural network model of sensoritopic maps with predictive short-term memory properties. Proc Natl Acad Sci USA 88:9653-9657.

Fenton AA, Csizmadia G, Muller RU. 2000a. Conjoint control of hippocampal place cell firing by two visual stimuli. I. The effects of moving the stimuli on firing field positions. J Gen Physiol 116:191-209.

Fenton AA, Csizmadia G, Muller RU. 2000b. Conjoint control of hippocampal place cell firing by two visual stimuli. II. A vector-field theory that predicts modifications of the representation of the envrionment. J Gen Physiol 116:211-221.

Fuhs MC, Touretzky DS. 2003. The mixture modeling theory of hippocampal place cell remapping. Soc Neurosci Abs 29:91.20.

Goodridge JP, Touretzky DS. 2000. Modeling attractor deformation in the rodent head direction system. J Neurophysiol, 83:3402-3410.

Hartley T, Burgess N, Lever C, Cacucci F, O’Keefe J. 2000. Modeling place fields in terms of the cortical inputs to the hippocampus. Hippocampus 10:369-379. 
Káli S, Dayan P. 2000. The involvement of recurrent connections in area CA3 in establishing the properties of place fields: a model. J Neurosci 20:7463-7477.

Knierim JJ. 2002. Dynamic interactions between local surface cues, distal landmarks, and intrinsic circuitry in hippocampal place cells. J Neurosci 22:6254-6264.

Lukashin AV, Amirikian BR, Mozhaev VL, Wilcox GL, Georgopoulos AP. 1996. Modeling motor cortical operations by an attractor network of stochastic neurons. Biol Cybern 74:255-261.

Muller RU, Kubie JL, Ranck JB Jr. 1987. Spatial firing patterns of hippocampal complex-spike cells in a fixed environment. J Neurosci 7:1935-1950.

O'Keefe J, Burgess N. 1996. Geometric determinants of the place fields of hippocampal neurons. Nature 381:425-428.

Olypher AV, Lansky P, Muller RU, Fenton AA. 2003. Quantifying location-specific information in the discharge of rat hippocampal place

Pinto DJ, Brumberg JC, Simons DJ, Ermentrout GB. 1996. A quantitative population model of whisker barrels: re-examining the WilsonCowan equations. J Comput Neurosci 3:247-264.

Pouget A, Zhang K, Deneve S, Latham PE. 1998. Statistically efficient estimation using population coding. Neural Comput 10:373-401.

Pouget A, Deneve S, Duhamel J-R. 2002. A computational perspective on multi-sensory spatial representations. Nat Rev Neurosci 3:741-747.

Redish AD. 1999. Beyond the cognitive map: from place cells to episodic memory. Cambridge, MA: MIT Press.

Redish AD, Elga AN, Touretzky DS. 1996. A coupled attractor model of the rodent head direction system. Network Comput Neural Syst 7:671-685. cells. J Neurosci Methods 127:123-135.

Redish AD, Touretzky DS. 1998. The role of the hippocampus in solving the Morris water maze. Neural Comput 10:73-111.

Samsonovich A, McNaughton BL. 1997. Path integration and cognitive mapping in a continuous attractor neural network model. J Neurosci 17:5900-5920.

Seung HS. 1996. How the brain keeps the eyes still. Proc Natl Acad Sci USA 93:13339-13344.

Shapiro ML, Tanila H, Eichenbaum H. 1997. Cues the hippocampal place cells encode: dynamic and hierarchcal representation of local and distal stimuli. Hippocampus 7:624-642.

Skaggs WE, Knierim JJ, Kudrimoti HS, McNaughton BL. 1995. A model of the neural basis of the rat's sense of direction. In: Tesauro G, Touretzky DS, Leen TK, editors. Advances in neural information processing systems. Vol 7. Cambridge, MA: MIT Press. 173180.

Tanila H, Shapiro ML, Eichenbaum H. 1997. Discordance of spatial representation in ensmbles of hippocampal place cells. Hippocampus 7:613-623.

Taube JS, Muller RI, Ranck JB Jr. 1990a. Head direction cells recorded from the postsubiculum in freely moving rats. II. Effects of environmental manipulations. J Neurosci 10:436-447.

Taube JS, Muller RI, Ranck JB Jr. 1990b. Head direction cells recorded from the postsubiculum in freely moving rats. I. Description and quantitative analysis. J Neurosci 10:420-435.

Zhang K. 1996. Representation of spatial orientation by the intrinsic dynamics of the head-direction cell ensemble: a theory. J Neurosci 16:2112-2126.

thresholding applied. $\mathrm{C}_{\mathrm{i}}$ is equal to the second summation in eq. (16). The variables $\mathrm{i}$ and $\mathrm{k}$ range over place cells and feature detectors, respectively. The expression count $(\mathrm{p})$ counts the number of place cells satisfying predicate $\mathrm{p}$ :

The feedforward inhibition term $\mathrm{I}_{\mathrm{FD}}$ produces a more focused and bump-like afferent input from the feature detectors to the place cells. It is calculated iteratively by making a large initial guess for $\mathrm{I}_{\mathrm{FD}}$ and then repeatedly reducing it by $10 \%$ until at least 10 grid cells receive feature detector activation of $\geq 0.4$. The quantity $\mathrm{B}_{\mathrm{i}}$ below denotes the feature detector afferent input for place cell $\mathrm{i}$, and $\mathrm{C}_{\mathrm{i}}$ is this afferent input with feedforward inhibition and
1. Calculate $\mathrm{B}_{\mathrm{i}}=\sum_{\mathrm{k}} \mathrm{w}_{\mathrm{ik}}^{\mathrm{EF}} \mathrm{FD}_{\mathrm{k}}$ for each place cell $\mathrm{i}$.

2. Set initial guess for the inhibition term $\mathrm{I}_{\mathrm{FD}}$ to $\max \left(\mathrm{B}_{\mathrm{i}}\right)-0.5$

3. Define $\mathrm{C}_{\mathrm{i}}\left(\mathrm{I}_{\mathrm{FD}}\right)$ as $\left[\mathrm{B}_{\mathrm{i}}-\mathrm{I}_{\mathrm{FD}}\right]_{+}$for each place cell $\mathrm{i}$.

4. While count $\mathrm{i}_{\mathrm{i}}\left(\mathrm{C}_{\mathrm{i}}\left(\mathrm{I}_{\mathrm{FD}}\right)>0.4\right)<10$ do $\mathrm{I}_{\mathrm{FD}} \leftarrow 0.9 \mathrm{I}_{\mathrm{FD}}$

5. Return $\mathrm{I}_{\mathrm{FD}}$ 